\title{
Skyrmion lattice with a giant topological Hall effect in a frustrated triangular-lattice magnet
}

\author{
Takashi Kurumaji1*, Taro Nakajima1, Max Hirschberger'1, Akiko Kikkawa ${ }^{1}$, Yuichi Yamasaki1,2,3, Hajime \\ Sagayama $^{4}$, Hironori Nakao ${ }^{4}$, Yasujiro Taguchi' ${ }^{1}$, Taka-hisa Arima ${ }^{1,5}$, Yoshinori Tokura ${ }^{1,6}$ \\ ${ }^{1}$ RIKEN Center for Emergent Matter Science (CEMS), Wako 351-0198, Japan. ${ }^{2}$ Research and Services Division of Materials Data and Integrated System (MaDIS), National \\ Institute for Materials Science (NIMS), Tsukuba 305-0047, Japan. ${ }^{3}$ PRESTO, Japan Science and Technology Agency (JST), Kawaguchi 332-0012, Japan. ${ }^{4}$ Institute of \\ Materials Structure Science, High Energy Accelerator Research Organization, Tsukuba, Ibaraki 305-0801, Japan. ${ }^{5}$ Department of Advanced Materials Science, The \\ University of Tokyo, Kashiwa 277-8561, Japan. ${ }^{6}$ Department of Applied Physics, The University of Tokyo, Tokyo 113-8656, Japan. \\ *Corresponding author. Email: takashi.kurumaji@riken.jp
}

Geometrically frustrated magnets can host complex spin textures, leading to unconventional electromagnetic responses. Magnetic frustration may also promote topologically nontrivial spin states such as magnetic skyrmions. Experimentally, however, skyrmions have largely been observed in noncentrosymmetric lattice structures or interfacial symmetry-breaking heterostructures. Here, we report the emergence of a Bloch-type skyrmion state in a frustrated centrosymmetric triangular-lattice magnet $\mathrm{Gd}_{2} \mathrm{PdSi}_{3}$. We observed a giant topological Hall response, indicating a field-induced skyrmion phase, which is further corroborated by the observation of in-plane spin modulation probed by resonant $x$ ray scattering. Our results may lead to further discoveries of emergent electrodynamics in magnetically frustrated centrosymmetric materials.

In geometrically frustrated magnets, where competing interactions among localized spins cannot be simultaneously satisfied, conventional magnetic orders are suppressed. Consequently, spins strongly fluctuate and can form a disordered state known as spin liquid state (1), or occasionally find a route to various spin textures, including spin spiral orders or more complex noncoplanar orders $(2,3)$. These spin states are mutually competing in energy, resulting in a complex magnetic phase diagram with respect to temperature, magnetic field, and pressure. An emerging spin state can be characterized from the perspective of geometrical correlation of spin vectors $\left(\boldsymbol{S}_{i}\right)$ on neighboring sites $(i, j, k)$ in a lattice. For example, the vector spin chirality $\boldsymbol{S}_{i} \times \boldsymbol{S}_{j}$ describes the handedness of a spin spiral (4), and the scalar spin chirality $\boldsymbol{S}_{i} \cdot\left(\boldsymbol{S}_{j} \times \boldsymbol{S}_{k}\right)$ is connected to time-reversal symmetry breaking $(5,6)$. These composite spin parameters couple with charge degrees of freedom in a correlated electron system, causing unconventional electromagnetic responses (7-10). Exploration of novel spin textures via magnetic frustration has been one of the central recent directions in condensed matter physics.

Spin configurations are characterized by topological numbers, which remain intact under local deformation or weak fluctuations (11). This concept has recently attracted growing interest since the discovery of magnetic skyrmion states in chiral magnets $(12,13)$. The magnetic skyrmion is a vortexlike nanometric spin structure carrying an integer topological number describing how many times magnetic moments within a skyrmion wrap a sphere (14). This quantization defines the particle nature of this spin texture with sensitivity to the electronic current and external electric/magnetic fields, highlighting the potential of magnetic skyrmions as information carriers (15). Extensive studies have successfully identified skyrmion-hosting materials in the form of both bulk compounds (16) and multilayer thin-film structures (17). From those, one can establish an empirical design principle for skyrmions $(18,19)$ : they appear in crystallographic lattice structures that lack inversion symmetry in or at the interfaces. These asymmetries cause the relativistic Dzyaloshinskii-Moriya (DM) interaction $(20,21)$, which inherently prefers twisted spin configurations. More recently, this dogma has been challenged in theories (22-24) that propose spontaneous symmetry breaking by stabilizing the skyrmion state in centrosymmetric lattices via magnetic frustration; however, experimental realization and observation of unconventional electronic responses have remained elusive.

Here we demonstrate that the metallic magnet $\mathrm{Gd}_{2} \mathrm{PdSi}_{3}$, composed of a triangular-lattice network of Gd atoms (Fig. 1A) in the centrosymmetric hexagonal structure, hosts a skyrmion-lattice (SkL) state upon the application of a magnetic field $(H)$ perpendicular to the triangular-lattice plane, which is robust down to the lowest measured temperature. The transition into the topological spin state is characterized by a prominent topological Hall response $(25,26)$, in sharp contrast to the adjacent magnetic phases. Using resonant x-ray scattering (RXS), we identify the long-range order of Gd spins modulated in the triangular lattice plane. The spin texture of 
the field-induced SkL phase is consistent with a triangularlattice of Bloch-type skyrmions (Fig. 1B).

$\mathrm{Gd}_{2} \mathrm{PdSi}_{3}$ belongs to a family of rare-earth intermetallics $R_{2} \mathrm{PdSi}_{3}$ (R: rare earth) (27). Its crystal structure derives from the simple $\mathrm{AlB}_{2}$-type structure with a triangular-lattice of $R$ atoms sandwiching a nonmagnetic honeycomb-lattice layer composed of Pd and Si atoms (Fig. 1A). Owing to the difference in atomic size, Si and Pd atoms order into a superstructure along both in- and out-of-plane directions (28), whereas the overall structure retains centrosymmetry (fig. S1A). This excludes the DM interaction as a source of the skyrmion state. Instead, the Ruderman-Kittel-Kasuya-Yosida (RKKY) type interaction among the local $4 f$ moments dominates (29-31); RKKY interactions on the triangular network of $4 f$ moments in $R_{2} \mathrm{PdSi}_{3}$ are moderately frustrated (32) and show rich magnetic phases including modulated structures (33). Specifically, in $\mathrm{Gd}_{2} \mathrm{PdSi}_{3}$ metamagnetic transitions have been observed under a magnetic field applied perpendicular to the triangular lattice, accompanied by nonmonotonic variations of longitudinal and transverse transport properties (34). These features suggest strong coupling between conduction electrons and Gd spins, and indicate that unconventional spin structures may emerge in the triangular-lattice network of Gd $4 f$ moments.

We first compare the magnetic phase diagram determined by the ac susceptibility ( $\chi^{\prime}$ ) for $H \| c$ in $\mathrm{Gd}_{2} \mathrm{PdSi}_{3}$ (Fig. 1C) with the contour mapping of the topological response of each phase probed by the topological Hall resistivity $\rho_{y x}^{\mathrm{T}}$ (Fig. 1D). Owing to the topological nature of skyrmions, they show characteristic emergent electrodynamic responses (14). In metallic materials, in particular, the scalar spin chirality of skyrmions acts like a fictitious magnetic field, which generates a transverse motion of electrons; this is known as the topological Hall effect (THE) $(25,26,35)$. The transverse resistivity $\rho_{y x}$ is generally composed of three components

$$
\rho_{y x}=R_{0} B+R_{S} M+\rho_{y x}^{T}
$$

where the first and the second terms are the normal and anomalous Hall resistivities proportional to the magnetic induction field $B$ and the magnetization $M$, respectively, and the third term represents the topological component. Because the first two terms can be determined from magnetization measurements, $\rho_{y x}^{\mathrm{T}}$ can be extracted reliably and is considered a good probe for the existence of skyrmions or related topological spin states in various materials (36). As shown in Fig. $1 \mathrm{C}$, peaks in $\chi{ }^{\prime}$ with respect to $H$ (fig. S2) define the phase boundaries for the three magnetic phases (IC-1, A, and IC-2) in addition to the paramagnetic (PM) state (34). In the $H$-T phase diagram, we overlay the contour plot of $\rho_{y x}^{\mathrm{T}}$ (Fig. 1D), which is deduced from the Hall resistivity measurements. The enhanced topological Hall signal appearing exclusively in the $A$ phase region suggests that in $\mathrm{Gd}_{2} \mathrm{PdSi}_{3}$ the application of $H$ induces topological phase transitions in the context of spin textures. The magnitude of the THE at the lowest temperature is as large as $2.6 \mu \Omega \mathrm{cm}$, which is one or two orders of magnitude larger than that in other skyrmion hosting materials such as $\mathrm{MnSi}(40 \mathrm{n} \Omega \mathrm{cm}$ under high pressure) $(25,26,35,37)$ and FeGe $(0.16 \mu \Omega \mathrm{cm}$ in a thin film) (38). This must be partly caused by a shorter wavelength of the spin modulation $(\sim 2.5 \mathrm{~nm})$ (fig. S5), which squeezes the emergent magnetic flux of a skyrmion, in contrast to the relatively large size of skyrmions $(10 \sim 100 \mathrm{~nm})$ in typical noncentrosymmetric (chiral or polar) magnets (36).

To corroborate the observation of the THE in the $A$ phase, we show a typical $\rho_{y x}-H$ curve together with the $M$ for $H \| c$ at $2 \mathrm{~K}$ (Fig. 2A). A sharp positive enhancement of $\rho_{y x}$ is apparent in the region between two stepwise changes of $M$ defining the first-order like transitions to and from the $A$ phase. In the IC-2 phase and higher field region, on the contrary, $\rho_{y x}$ stays negative with nearly field-linear behavior, at least up to $140 \mathrm{kOe}$ (fig. S3A), where $M$ is $13.7 \mu_{\mathrm{B}} /$ f.u. approaching the saturation value expected for the value of local Gd moment. This nearly saturated phase in principle hosts a topologically trivial spin arrangement, allowing us to describe the Hall response with the first two terms in Eq. 1. The black solid line in Fig. 2A shows the fit to the high-field data of $\rho_{y x}$. The fitting quality is excellent for all measured temperatures (fig. S3A), which allows us to unambiguously extract $\rho_{y x}^{\mathrm{T}}$ from $\rho_{y x}$ (Figs. 2B and $1 \mathrm{C}$ ). We note that the quality of the fit is little affected by using a different formula, e.g., assuming skew scattering type anomalous Hall effect (fig. S4). Figure 2C shows the evolution of the peak in $\rho_{y x}^{\mathrm{T}}$ with temperature. Continuous decrease of $\rho_{y x}^{\mathrm{T}}$ toward zero around $20 \mathrm{~K}$ suggests that this response is affected by the magnitude of the molecular field from $4 f$ moment on the conduction electron through an $f$ - $d$ coupling, consistent with the scalar spin chirality model for the THE (35). We note that the effective magnetic field $\left(B_{\text {eff }}\right)$ for the maximum $\rho_{y x}^{\mathrm{T}}$ is around $-39 \mathrm{~T}(39)$, which is a factor of $0.07(\equiv P)$ smaller than the bare emergent magnetic field $\left(B_{\mathrm{em}} \sim-570 \mathrm{~T}\right)$ estimated from the skyrmion density. The polarization factor $P$ is one order of magnitude smaller than those in $\mathrm{MnSi}$ under pressure $(P \sim$ $0.25-0.38)$ and slightly-doped $\mathrm{Mn}_{1-x} \mathrm{Fe}_{x} \mathrm{Si}(P \sim 0.3-0.45)$ (37). This may be caused by the moderate $f$ - $d$ coupling in the present rare earth system as compared with the strong $d-d$ coupling in transition metal compounds.

To further examine the nature of the SkL state in the $A$ phase, we present the Hall resistivity as a function of the angle between $H$ and the $c$ axis in the experimental 
configuration illustrated in the inset of Fig. 2D. At $\phi=0^{\circ}(H \| c)$ with $H=9.9 \mathrm{kOe}$ in the $A$ phase, $\rho_{y x}$ starts from a large positive value. As $H$ rotates clockwise away from the $c$ axis, the value of $\rho_{y x}$ remains flat until it experiences an abrupt drop to near zero at around $\phi=45^{\circ}$. A hysteresis with the width of $\sim 15^{\circ}$ is observed between clockwise and counter clockwise rotation scans of $H$, pointing to the firstorder nature of this $H$-direction sensitive phase transition. This should be compared to thin film systems (40,41), where the SkL is confined in a two-dimensional space, and survives only in an $H$ oriented nearly perpendicular to the lattice plane. Similar behavior may be expected for the present system composed of stacked triangular-lattice layers. The above observation provides a measure of the topological number for the spin texture where the topological Hall signal sharply transitions from finite to zero upon the destabilization of the SkL state. In contrast, at $H=40 \mathrm{kOe}$ far above the upper critical field of the $A$ phase, a smooth evolution of $\rho_{y x}$ is observed with negligible hysteresis. This high-field $\rho_{y x}$, whose absolute magnitude is much smaller than the SkL signal, follows $\cos \phi$ (black solid line in Fig. 2D), indicating that $M$ closely follows the rotating $H$ and that the projection of $M$ and $B$ to the $c$ axis produce the first two terms in Eq. 1 as dominant contributions to $\rho_{y x}$ outside the $A$ phase region.

Having identified the emergence of a topological electromagnetic response in the $A$ phase, we examined the Gd spin structure under $H$ along the $c$ axis by means of the magnetic RXS in resonance with the Gd $L_{2}$ edge. We observed the magnetic modulation along in-plane directions represented by the reciprocal-space vector $\boldsymbol{Q}_{1}=(q, 0,0)$ (and equivalent $\boldsymbol{Q}_{2}$ $=(0,-q, 0)$ and $\left.\boldsymbol{Q}_{3}=(q,-q, 0)\right)$, in the magnetically ordered phase (39). Here, $q$ ( $\sim 0.14$ r.l.u.) is the magnetic modulation wavenumber. In Fig. 3, A and B, we show $M$ and $q$, respectively, as a function of $H$, which is applied along the $c$ axis; the data were taken at $5 \mathrm{~K}$. To define the phase boundary for each phase, we show the difference $\triangle M$ between the measurements of $M$ for the $H$-increasing and decreasing scans (Fig. $3 \mathrm{~A})$. In the IC- 1 phase, $q$ is almost independent of $H$, and starts to gradually increase on entering the $A$ phase and furthermore in the IC-2 state. Despite the clear first-order nature for each transition (vertical gray lines), $q$ shows merely a weak kink at each phase boundary and changes only $4 \%$ in total between 0 Oe in the IC- 1 phase and $20 \mathrm{kOe}$ in the IC-2 phase. The orientation of the $Q$ vectors with respect to the triangular lattice does not change across these metamagnetic transitions. This restricts the candidate spin textures for each phase to the spin modulations with one or several equivalent $Q$ vectors plus a component of homogeneous magnetization $(q=0)$ along the $c$ axis. This is consistent with the intermediate-field SkL state which can be seen as a superposition of three spiral spin modulations with their magnetic modulation vectors lying in the triangular-lattice plane and pointing $120^{\circ}$ away from each other.

Figure 3C shows the $H$ dependence of the scattering intensities for respective satellite peaks for the three $Q$ vectors measured around a Bragg spot $(2,2,0)$ in the $H$-decreasing scan. Starting from the high-field IC-2 phase region (10 kOe $<H<20 \mathrm{kOe}$ ), we observed that the intensity for one of the $Q$ vectors $\left(I_{\mathbf{Q} 2}\right)$ is markedly weak compared with $I_{\mathbf{Q} 1}$ and $I_{\mathbf{Q} 3}$. A fan-like structure (fig. S7A) provides a good explanation for this feature as follows. Polarization analysis of the scattered $\mathrm{x}$-ray, which enables to decompose the in-plane $\left(\boldsymbol{m}_{\perp}\right)$ and out-of-plane $\left(m_{z}\right)$ components of the modulated magnetic moment (39), reveals the negligibly-weak modulating $m_{z}$ component (fig. S6B) for the magnetic structure of the IC-2 state. We thus propose that a possible magnetic structure for the IC-2 state is a fan-like or a transverse conical structure (fig. S7, A and B), both of which are lacking global scalar spin chirality in accord with the absence of a topological contribution in $\rho_{y x}$. Of the two proposed magnetic structures, the fan model gives a better fit to the observed intensity, although both fit show deviations from experiment. The observed imbalance of the scattering intensity among the three $Q$-domains is suggestive of the single- $Q$ nature of this phase, and stems perhaps from residual strains on the sample induced by shaping and attaching it on the sample holder (42).

With decreasing $H$ (Fig. 3C), the intensities for all the three $Q$ vectors show a step-wise increase upon entering the $A$ phase. Such a simultaneous increase of intensity for every $Q$ is associated with the developing $m_{z}$ (modulation component) as shown in Fig. 3D, which is absent in the IC-2 phase. This fact points to a noncoplanar spin texture in the topological $A$ phase. Further decreasing $H$ (Fig. 3C), the intensity for each $Q$ vector is almost unchanged while a prominent peak in $\triangle M$ (Fig. 3A) suggests a first-order phase transition from the $A$ phase to the IC- 1 phase. The polarization analysis reveals the presence of an $m_{z}$ component (fig. S6A) comparable with that of the $A$ phase, suggesting a similarity of the spin configurations for both phases.

Looking back to the polarization analysis for the $A$ phase (Fig. 3D), the intensity $I_{\pi-\pi^{\prime}}$ for the $\pi-\pi^{\prime}$ channel $\left(\propto m_{\mathrm{z}}^{2}\right)$ is of almost the same magnitude for all $\boldsymbol{Q}_{i}$, consistent with the triple- $Q$ nature of the skyrmion state. $I_{\pi-\sigma^{\prime}}\left(\propto\left(\boldsymbol{m}_{\perp} \cdot \boldsymbol{k}_{\mathrm{i}}\right)^{2}\right)$ is, on the other hand, correlated with $\boldsymbol{m}_{\perp}$ to show clear $\boldsymbol{Q}_{i}$ dependence. For the Bloch-type SkL state, the spin texture is composed of a superposition of the three proper-screw spin modulations (Fig. 3E), where $\boldsymbol{m}_{\perp}$ is perpendicular to each $\boldsymbol{Q}_{i}$ vector (Fig. 3E, inset). As shown in Fig. $3 \mathrm{~F}$ and the inset, the 
direction of $\boldsymbol{Q}_{2}$ is particularly closer to $\boldsymbol{k}_{\mathrm{i}}$ than $\boldsymbol{Q}_{1}$ and $\boldsymbol{Q}_{3}$ are, i.e., the direction of $\boldsymbol{m}_{\perp}$ for $\boldsymbol{Q}_{2}$ is closer to the direction normal to $\boldsymbol{k}_{\mathrm{i}}$ than those for $\boldsymbol{Q}_{1}$ and $\boldsymbol{Q}_{3}$. This feature is consistent with the $\boldsymbol{Q}$ dependence of $I_{\pi-\sigma^{\prime}}$. Furthermore, a quantitative comparison between the calculated and observed intensities reveals that the magnetic structures in the $A$ phase can be reproduced by hybridization of the three proper screws with equivalent amplitude plus the uniform moment along $z$ (fig. S6C), being consistent with the picture of the Bloch-type SkL state. We note that this spin texture spontaneously breaks the inversion symmetry and potentially hosts domains for handedness of skyrmions. Preference for the Bloch-type spin configuration over the Néel or the antiskyrmion types ones is consistent with the effect of the dipole-dipole interaction (14, 43), which is generally significant in Gd compounds.

The scattering intensities in the IC-1 state (fig. S6, A and C) suggest that the IC-1 state may also be of triple- $Q$ nature as well but forms a spin texture topologically distinct from that of the $A$ phase. We note that there remains a degree of freedom for the phase $\left(\varphi_{i}\right)$ among the three helical modulations (39). When the $\varphi_{i}$ for each $Q_{i}$-vector is $0(\bmod 2 \pi)$, the triple- $Q$ state is equivalent to the Bloch-type SkL state as exemplified by the $A$ phase here. For $\varphi_{i}=\pi / 6$, the triple- $Q$ state is composed of a triangular-lattice of merons and antimerons (fig. S7C) with no net scalar spin chirality at zero field; this is compatible with the observed features for the IC-1 state (39). The possible emergence of a triple- $Q$ zero-field ground state (IC-1) may be an interesting difference from the conventional nonocentrosymmetric skyrmion-hosting systems, which typically show a single- $Q$ helical state as the zero-field state (36). We also note unconventional features beyond the conventional helical or conical state in the IC-1 phase. As shown in Fig. 2B, $\rho_{y x}^{\mathrm{T}}$ starts to gradually increase from zero field prior to a steep increase characterizing the transition to the skyrmion state, which can be explained by the proposed non-coplanar nature in the IC-1 state: the meron-antimeron lattice can show an $H$-induced scalar spin chirality (39).

According to existing theories, skyrmion phase down to the lowest temperature is enabled by the magnetic frustration with support from additional effects, such as magnetic anisotropy owing to the spin-orbit coupling $(23,44)$ and higher-order RKKY-like interaction (24). Interestingly, it is predicted that the latter mechanism can stabilize a zero-field multiple- $Q$ state (albeit not identical with the present IC-1 state) (45), suggesting that nearly degenerate multiple- $Q$ orders may exist in the ground state of the RKKY-based intermetallics. We observe that the magnetic structure for the IC1 phase shows a certain ellipticity of the spin spiral form (fig. S9), which is suggestive of weak easy-plane anisotropy playing some role to stabilize the IC-1 state.
In addition to the enhanced topological Hall effect, it has been theoretically predicted that the skyrmion in a centrosymmetric lattice shows unique properties such as the compatible formation of antiskyrmion with skyrmion $(22,23)$ and the helicity dependent current responses $(43,44)$. These properties provide the skyrmions as individual particles with internal degrees of freedom, which are absent in noncentrosymmetric systems with innate chirality or polarity. The conduction-electron mediated competing magnetic interactions on a geometrically frustrated lattice will provide a platform for emergent electrodynamics owing to topological spin textures and will provide a link between the concepts of spin topology and magnetic frustration.

\section{REFERENCES AND NOTES}

1. L. Balents, Spin liquids in frustrated magnets. Nature 464, 199-208 (2010). doi:10.1038/nature08917 Medline

2. H. T. Diep, Frustrated Spin Systems (World Scientific, Singapore, 2004).

3. C. Lacroix, P. Mendels, F. Mila, Introduction to Frustrated Magnetism (Springer Series in Solid State Sciences, Vol. 164, Springer, 2011).

4. V. Simonet, M. Loire, R. Ballou, Magnetic chirality as probed by neutron scattering. Eur. Phys. J. Spec. Top. 213, 5-36 (2012). doi:10.1140/epjst/e2012-01661-8

5. V. Kalmeyer, R. B. Laughlin, Equivalence of the resonating-valence-bond and fractional quantum Hall states. Phys. Rev. Lett. 59, 2095-2098 (1987). doi:10.1103/PhysRevLett.59.2095 Medline

6. X. G. Wen, F. Wilczek, A. Zee, Chiral spin states and superconductivity. Phys. Rev. B 39, 11413-11423 (1989). doi:10.1103/PhysRevB.39.11413 Medline

7. S.-W. Cheong, M. Mostovoy, Multiferroics: A magnetic twist for ferroelectricity. Nat. Mater. 6, 13-20 (2007). doi:10.1038/nmat1804 Medline

8. Y. Taguchi, Y. Oohara, H. Yoshizawa, N. Nagaosa, Y. Tokura, Spin chirality, Berry phase, and anomalous Hall effect in a frustrated ferromagnet. Science 291, 25732576 (2001). doi:10.1126/science.1058161 Medline

9. Y. Machida, S. Nakatsuji, S. Onoda, T. Tayama, T. Sakakibara, Time-reversal symmetry breaking and spontaneous Hall effect without magnetic dipole order. Nature 463, 210-213 (2010). doi:10.1038/nature08680 Medline

10. C. D. Batista, S.-Z. Lin, S. Hayami, Y. Kamiya, Frustration and chiral orderings in correlated electron systems. Rep. Prog. Phys. 79, 084504 (2016). doi:10.1088/0034-4885/79/8/084504 Medline

11. H. B. Braun, Topological effects in nanomagnetism: From superparamagnetism to chiral quantum solitons. Adv. Phys. 61, 1-116 (2012). doi:10.1080/00018732.2012.663070

12. S. Mühlbauer, B. Binz, F. Jonietz, C. Pfleiderer, A. Rosch, A. Neubauer, R. Georgii, P. Böni, Skyrmion lattice in a chiral magnet. Science 323, 915-919 (2009). doi:10.1126/science.1166767 Medline

13. X. Z. Yu, Y. Onose, N. Kanazawa, J. H. Park, J. H. Han, Y. Matsui, N. Nagaosa, Y. Tokura, Real-space observation of a two-dimensional skyrmion crystal. Nature 465, 901-904 (2010). doi:10.1038/nature09124 Medline

14. N. Nagaosa, Y. Tokura, Topological properties and dynamics of magnetic skyrmions. Nat. Nanotechnol. 8, 899-911 (2013). doi:10.1038/nnano.2013.243 Medline

15. A. Fert, V. Cros, J. Sampaio, Skyrmions on the track. Nat. Nanotechnol. 8, 152-156 (2013). doi:10.1038/nnano.2013.29 Medline

16. A. Bauer, C. Pfleiderer, Generic Aspects of Skyrmion Lattices in Chiral Magnets (Springer International Publishing, 2016).

17. W. Jiang, G. Chen, K. Liu, J. Zang, S. G. E. te Velthuis, A. Hoffmann, Skyrmions in magnetic multilayers. Phys. Rep. 704, 1-49 (2017). doi:10.1016/i.physrep.2017.08.001

18. A. N. Bogdanov, D. A. Yablonskii, Sov. Phys. JETP 68, 101 (1989).

19. A. Bogdanov, A. Hubert, Thermodynamically stable magnetic vortex states in magnetic crystals. J. Magn. Magn. Mater. 138, 255-269 (1994). doi:10.1016/0304-8853(94)90046-9

20. I. Dzyaloshinskii, A thermodynamic theory of "weak" ferromagnetism of 
antiferromagnetics. J. Phys. Chem. Solids 4, 241-255 (1958). doi:10.1016/00223697(58)90076-3

21. T. Moriya, Anisotropic Superexchange Interaction and Weak Ferromagnetism. Phys. Rev. 120, 91-98 (1960). doi:10.1103/PhysRev.120.91

22. T. Okubo, S. Chung, H. Kawamura, Multiple-q states and the Skyrmion lattice of the triangular-lattice Heisenberg antiferromagnet under magnetic fields. Phys. Rev. Lett. 108, 017206 (2012). doi:10.1103/PhysRevLett.108.017206 Medline

23. A. O. Leonov, M. Mostovoy, Multiply periodic states and isolated skyrmions in an anisotropic frustrated magnet. Nat. Commun. 6, 8275 (2015). doi:10.1038/ncomms9275 Medline

24. S. Hayami, R. Ozawa, Y. Motome, Effective bilinear-biquadratic model for noncoplanar ordering in itinerant magnets. Phys. Rev. B 95, 224424 (2017). doi:10.1103/PhysRevB.95.224424

25. M. Lee, W. Kang, Y. Onose, Y. Tokura, N. P. Ong, Unusual Hall effect anomaly in MnSi under pressure. Phys. Rev. Lett. 102, 186601 (2009). doi:10.1103/PhysRevLett.102.186601 Medline

26. A. Neubauer, C. Pfleiderer, B. Binz, A. Rosch, R. Ritz, P. G. Niklowitz, P. Böni, Topological Hall effect in the A phase of MnSi. Phys. Rev. Lett. 102, 186602 (2009). doi:10.1103/PhysRevLett.102.186602 Medline

27. P. A. Kotsanidis, J. K. Yakinthos, E. Gamari-Seale, Magnetic properties of the ternary rare earth silicides $\mathrm{R}_{2} \mathrm{PdSi}_{3}(\mathrm{R}=\mathrm{Pr}, \mathrm{Nd}, \mathrm{Gd}, \mathrm{Tb}, \mathrm{Dy}, \mathrm{Ho}, \mathrm{Er}, \mathrm{Tm}$ and $\mathrm{Y})$. J. Magn. Magn. Mater. 87, 199-204 (1990). doi:10.1016/0304-8853(90)90215-C

28. F. Tang, M. Frontzek, J. Dshemuchadse, T. Leisegang, M. Zschornak, R. Mietrach, J.-U. Hoffmann, W. Löser, S. Gemming, D. C. Meyer, M. Loewenhaupt, Crystallographic superstructure in $\mathrm{R} 2 \mathrm{PdSi} 3$ compounds ( $\mathrm{R}=$ heavy rare earth). Phys. Rev. B 84, 104105 (2011). doi:10.1103/PhysRevB.84.104105

29. M. A. Ruderman, C. Kittel, Indirect Exchange Coupling of Nuclear Magnetic Moments by Conduction Electrons. Phys. Rev. 96, 99-102 (1954). doi:10.1103/PhysRev.96.99

30. T. Kasuya, A Theory of Metallic Ferro- and Antiferromagnetism on Zener's Model. Prog. Theor. Phys. 16, 45-57 (1956). doi:10.1143/PTP.16.45

31. K. Yosida, Magnetic Properties of Cu-Mn Alloys. Phys. Rev. 106, 893-898 (1957). doi:10.1103/PhysRev.106.893

32. D. S. Inosov, D. V. Evtushinsky, A. Koitzsch, V. B. Zabolotnyy, S. V. Borisenko, A. A. Kordyuk, M. Frontzek, M. Loewenhaupt, W. Löser, I. Mazilu, H. Bitterlich, G. Behr, J.-U. Hoffmann, R. Follath, B. Büchner, Electronic structure and nesting-driven enhancement of the RKKY interaction at the magnetic ordering propagation vector in $\mathrm{Gd}_{2} \mathrm{PdSi}_{3}$ and $\mathrm{Tb}_{2} \mathrm{PdSi}_{3}$. Phys. Rev. Lett. 102, 046401 (2009). doi:10.1103/PhysRevLett.102.046401 Medline

33. A. Szytuła, M. Hofmann, B. Penc, M. Ślaski, S. Majumdar, E. V. Sampathkumaran, A. Zygmunt, Magnetic behaviour of $\mathrm{R}_{2} \mathrm{PdSi} 3$ compounds with $\mathrm{R}=\mathrm{Ce}, \mathrm{Nd}, \mathrm{Tb}-\mathrm{Er}$. J. Magn. Magn. Mater. 202, 365-375 (1999). doi:10.1016/S0304-8853(99)004102

34. S. R. Saha, H. Sugawara, T. D. Matsuda, H. Sato, R. Mallik, E. V. Sampathkumaran, Magnetic anisotropy, first-order-like metamagnetic transitions, and large negative magnetoresistance in single-crystal $\mathrm{Gd}_{2} \mathrm{PdSi}_{3}$. Phys. Rev. B 60, $12162-$ 12165 (1999). doi:10.1103/PhysRevB.60.12162

35. R. Ritz, M. Halder, C. Franz, A. Bauer, M. Wagner, R. Bamler, A. Rosch, C. Pfleiderer, Giant generic topological Hall resistivity of MnSi under pressure. Phys. Rev. B 87, 134424 (2013). doi:10.1103/PhysRevB.87.134424

36. N. Kanazawa, S. Seki, Y. Tokura, Noncentrosymmetric Magnets Hosting Magnetic Skyrmions. Adv. Mater. 29, 1603227 (2017). doi:10.1002/adma.201603227 Medline

37. B. J. Chapman, M. G. Grossnickle, T. Wolf, M. Lee, Large enhancement of emergent magnetic fields in MnSi with impurities and pressure. Phys. Rev. B 88, 214406 (2013). doi:10.1103/PhysRevB.88.214406

38. S. X. Huang, C. L. Chien, Extended Skyrmion phase in epitaxial FeGe(111) thin films. Phys. Rev. Lett. 108, 267201 (2012). doi:10.1103/PhysRevLett.108.267201 Medline

39. See materials and methods and supplementary text in the supplementary materials.

40. T. Yokouchi, N. Kanazawa, A. Tsukazaki, Y. Kozuka, M. Kawasaki, M. Ichikawa, F. Kagawa, Y. Tokura, Stability of two-dimensional skyrmions in thin films of $\mathrm{Mn}_{1-\mathrm{F}} \mathrm{Fe}$-Si investigated by the topological Hall effect. Phys. Rev. B 89, 064416 (2014). doi:10.1103/PhysRevB.89.064416
41. Y. Ohuchi, Y. Kozuka, M. Uchida, K. Ueno, A. Tsukazaki, M. Kawasaki, Topological Hall effect in thin films of the Heisenberg ferromagnet EuO. Phys. Rev. B 91, 245115 (2015). doi:10.1103/PhysRevB.91.245115

42. T. Inami, N. Terada, H. Kitazawa, O. Sakai, Resonant Magnetic X-ray Diffraction Study on the Triangular Lattice Antiferromagnet $\mathrm{GdPd}_{2} \mathrm{Al}_{3}$. J. Phys. Soc. Jpn. 78 084713 (2009). doi:10.1143/JPS J.78.084713

43. X. Zhang, J. Xia, Y. Zhou, X. Liu, H. Zhang, M. Ezawa, Skyrmion dynamics in a frustrated ferromagnetic film and current-induced helicity locking-unlocking transition. Nat. Commun. 8, 1717 (2017). doi:10.1038/s41467-017-01785-w Medline

44. S.-Z. Lin, S. Hayami, Ginzburg-Landau theory for skyrmions in inversionsymmetric magnets with competing interactions. Phys. Rev. B 93, 064430 (2016). doi:10.1103/PhysRevB.93.064430

45. R. Ozawa, S. Hayami, Y. Motome, Zero-Field Skyrmions with a High Topological Number in Itinerant Magnets. Phys. Rev. Lett. 118, 147205 (2017). doi:10.1103/PhysRevLett.118.147205 Medline

46. T. Kurumaji et al., Skyrmion in frustrated triangular-lattice, Version 1.0, Zenodo (2019); http://doi.org/10.5281/zenodo.3240669.

47. Y. Xu, W. Löser, Y. Guo, X. Zhao, L. Liu, Crystal growth of $\mathrm{Gd}_{2} P d \mathrm{PSi}_{3}$ intermetallic compound. Trans. Nonferrous Met. Soc. China 24, 115-119 (2014). doi:10.1016/S1003-6326(14)63035-1

48. M. Lee, Y. Onose, Y. Tokura, N. P. Ong, Hidden constant in the anomalous Hall effect of high-purity magnet MnSi. Phys. Rev. B 75, 172403 (2007). doi:10.1103/PhysRevB.75.172403

49. N. Nagaosa, J. Sinova, S. Onoda, A. H. MacDonald, N. P. Ong, Anomalous Hall effect. Rev. Mod. Phys. 82, 1539-1592 (2010). doi:10.1103/RevModPhys.82.1539

50. F. Tang, P. Link, M. Frontzek, J.-M. Mignot, J.-U. Hoffmann, W. Löser, M. Loewenhaupt, Neutron diffraction study of magnetic structures in single crystal $\mathrm{Ho}_{2} \mathrm{PdSi}_{3}$ in magnetic fields up to 5 T. J. Phys. Conf. Ser. 251, 012017 (2010). doi:10.1088/1742-6596/251/1/012017

51. M. Frontzek, A. Kreyssig, M. Doerr, A. Schneidewind, J.-U. Hoffmann, M. Loewenhaupt, Frustration in $\mathrm{R}_{2} \mathrm{PdSi}{ }_{3}(\mathrm{R}=\mathrm{Tb}, \mathrm{Er})$ compounds: Spin-glass or magnetic short range order? Neutron diffraction studies. J. Phys. Condens. Matter 19, 145276 (2007). doi:10.1088/0953-8984/19/14/145276

52. R. Mallik, E. V. Sampathkumaran, M. Strecker, G. Wortmann, Observation of a minimum in the temperature-dependent electrical resistance above the magnetic-ordering temperature in $\mathrm{Gd}_{2} \mathrm{PdSi}_{3}$. Europhys. Lett. 41, 315-320 (1998). doi:10.1209/ep//i1998-00149-4

53. M. Blume, in Resonant Anomalous X-Ray Scattering, G. Materlik, C. J. Sparks, K. Fisher, Eds. (Elsevier, Amsterdam, 1994), p. 495.

54. B. Binz, A. Vishwanath, Chirality induced anomalous-Hall effect in helical spin crystals. Physica B 403, 1336-1340 (2008). doi:10.1016/j.physb.2007.10.136

\section{ACKNOWLEDGMENTS}

The authors thank X. Z. Yu for efforts on Lorentz transmission electron microscopy experiments, and L. Ye, K. S. Okada, S. Hayami, H. Ishizuka, Y. Motome, and N. Nagaosa for enlightening discussions. Funding: $X$-ray scattering measurements were performed under the approval of the Photon Factory Program Advisory Committee (Proposals No. 2015S2-007) at the Institute of Material Structure Science, High Energy Accelerator Research Organization (KEK). This research was supported in part by Grant-in-Aid for Scientific Research(S) No. 24224009 , Grant-In-Aid for Young Scientists(B) No. 17K14351, Grant-in-Aid for Scientific Research No. $16 \mathrm{H} 05990$ from the Japan Society for the Promotion of Science (JSPS), and by PRESTO No. JPMJPR177A from Japan Science and Technology Agency (JST). One of the authors, M.H., was supported as a JSPS international research fellow. Author contributions: T.K. and Y.Tokura conceived the project. T.K. A.K., and Y.Taguchi grew the single crystals of $\mathrm{Gd}_{2} \mathrm{PdSi}_{3}$. T.K. characterized the magnetic and transport properties of the samples with the help of M.H., and A.K. T.N., Y.Y., H.S., H.N., and T.A performed the resonant x-ray diffraction measurement. T.K., T.N., M.H, T.A., and Y.Tokura jointly discussed the result and wrote the manuscript with contributions from all authors. Competing interests: The authors declare no competing interests. Data and materials availability: Data reported in this paper are archived online (46). 


\section{SUPPLEMENTARY MATERIALS}

science.sciencemag.org/cgi/content/full/science.aau0968/DC1

Materials and Methods

Supplementary Text

Fig. S1 to S9

References (47-54)

7 May 2018; accepted 30 July 2019

Published online 8 August 2019

10.1126/science.aau0968

Fig. 1. Phase diagram and topological Hall effect in $\mathrm{Gd}_{2} \mathrm{PdSi}_{3}$. (A) The basic AlB $\mathrm{Al}_{2}$-type crystal structure for $\mathrm{Gd}_{2} \mathrm{PdSi}_{3}$. (B) The illustration of the spin texture in the skyrmion lattice (SkL) state. Each arrow indicates a magnetic moment at each $\mathrm{Gd}$ site. (C and D) The contour plot of (C) $\chi^{\prime}$ and (D) $\rho_{y x}^{\mathrm{T}}$ (see the text for the definition) for $H \| c$. "A" represents the SkL phase and "PM" the paramagnetic phase. The "IC-1" and "IC-2" denote incommensurate spin state phases in near-zero and high-field regions, respectively. Circular (triangular) symbols were determined by a peak or a kink in $x^{\prime}-H\left(x^{\prime}-T\right)$ scan (see fig. S2).
A

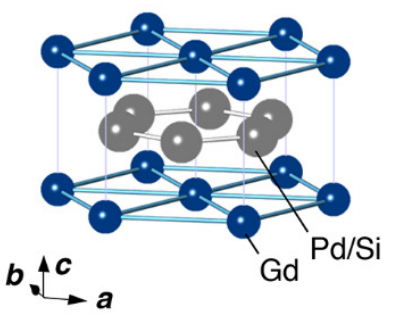

B

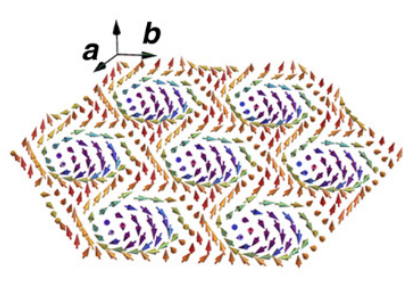

C

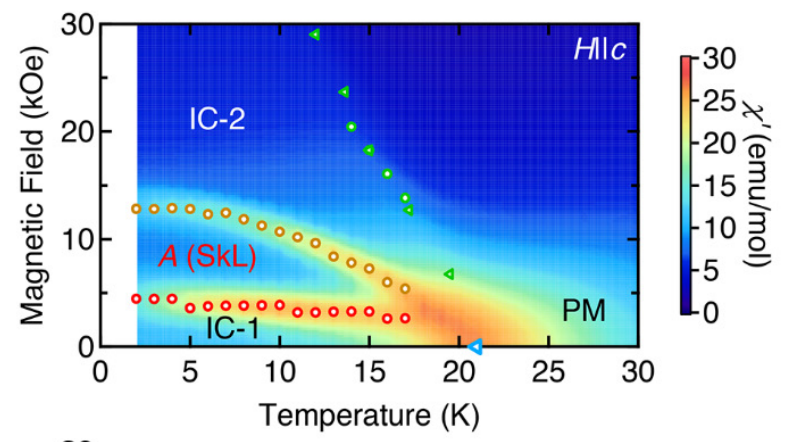

D

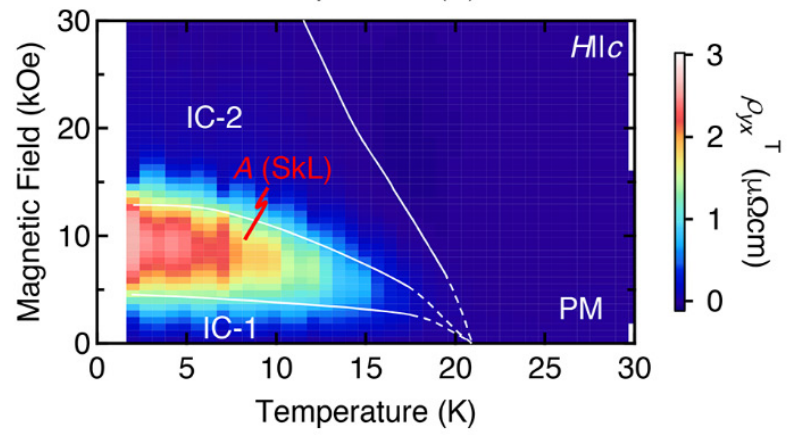



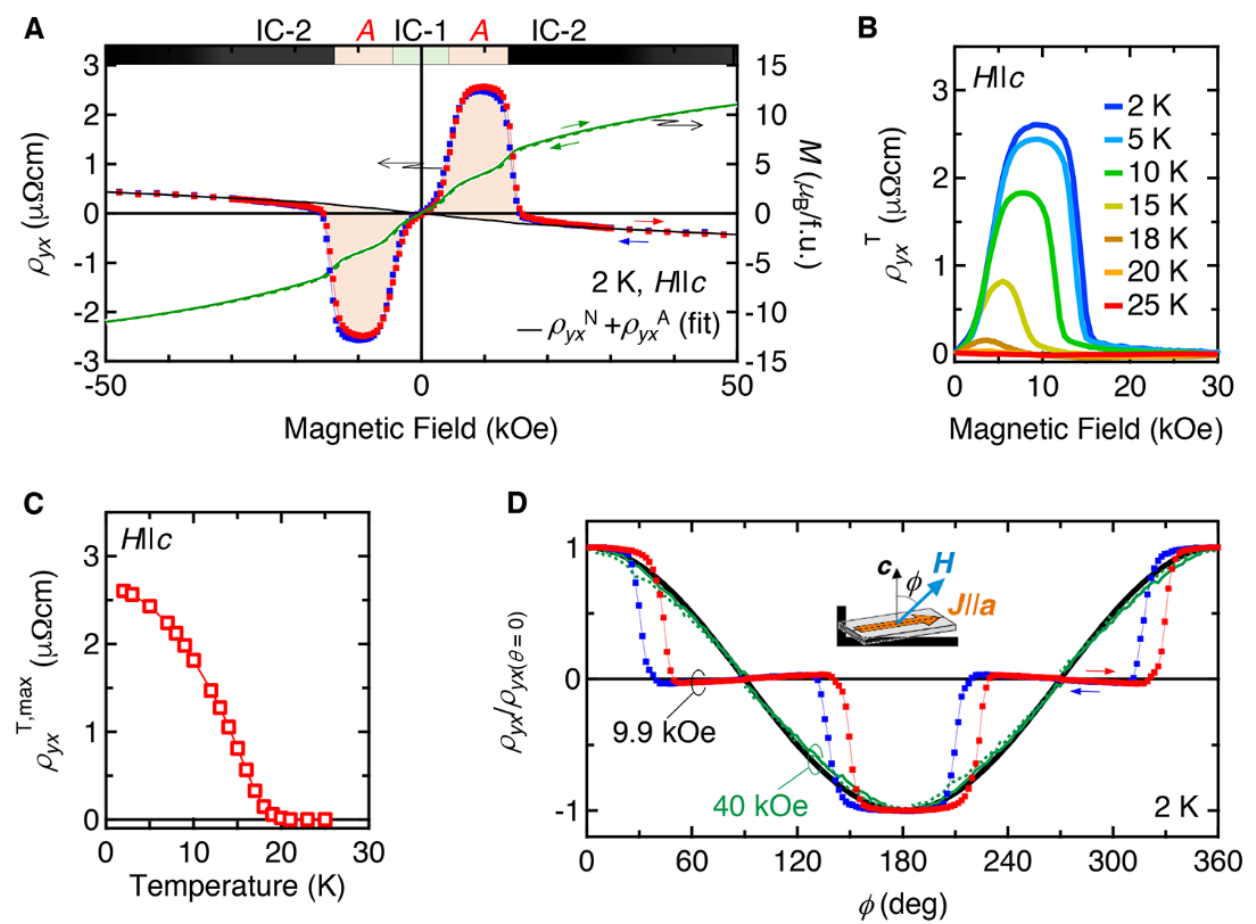

Fig. 2. Temperature and angular dependence of the topological Hall effect in $\mathrm{Gd}_{2} \mathrm{PdSi}_{3}$. (A) $H$ dependence of $\rho_{y x}$ (left axis) and $M$ (right axis) for $H \| c$ at $2 \mathrm{~K}$. Red (blue) curve is for the $H$-increasing (-decreasing) scan. The black curve indicates the sum of the normal $\left(\rho_{y x}^{\mathrm{N}}\right)$ and the anomalous $\left(\rho_{y x}^{\mathrm{A}}\right)$ components of Hall resistivity. (B) $H$ dependence of topological Hall component $\rho_{y x}^{\mathrm{T}}$ at various temperatures. (C) Temperature dependence of the maximum values of $\rho_{y x}^{\mathrm{T}}\left(\rho_{y x}^{\mathrm{T}, \max }\right)$. (D) Normalized transverse resistivity at $2 \mathrm{~K}$ with $\mathrm{H}$ rotating in the ac plane. Red (blue) symbols and green solid (dashed) line are in a (counter-) clockwise rotation. Inset defines the rotation angle $\phi$. The reference line $\cos \phi$ is shown by the black solid line. 


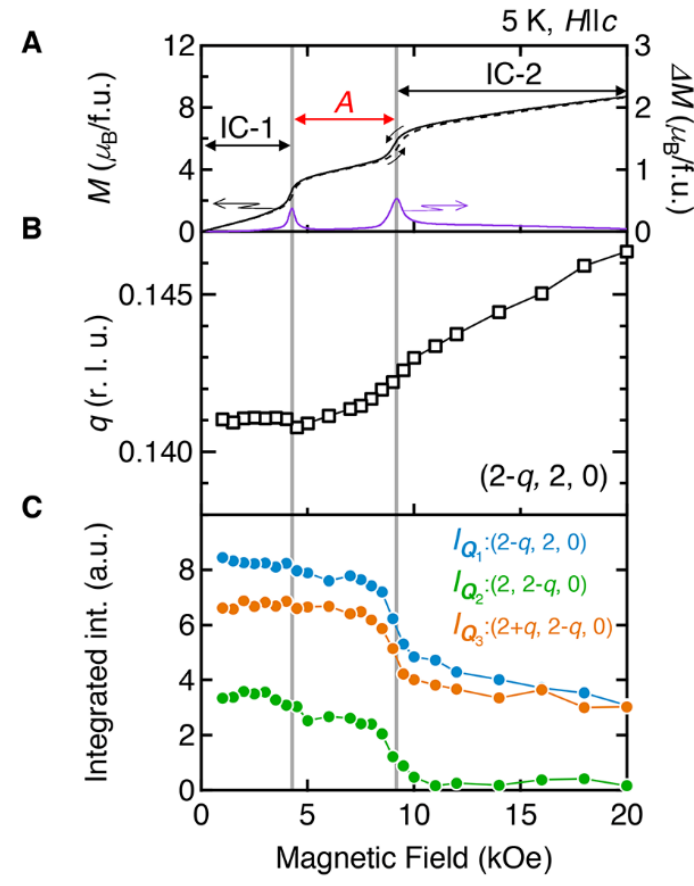

D

$(2,2,0)+/-Q_{i}, 5 \mathrm{~K}, 7 \mathrm{kOe}, A(\mathrm{SkL})$
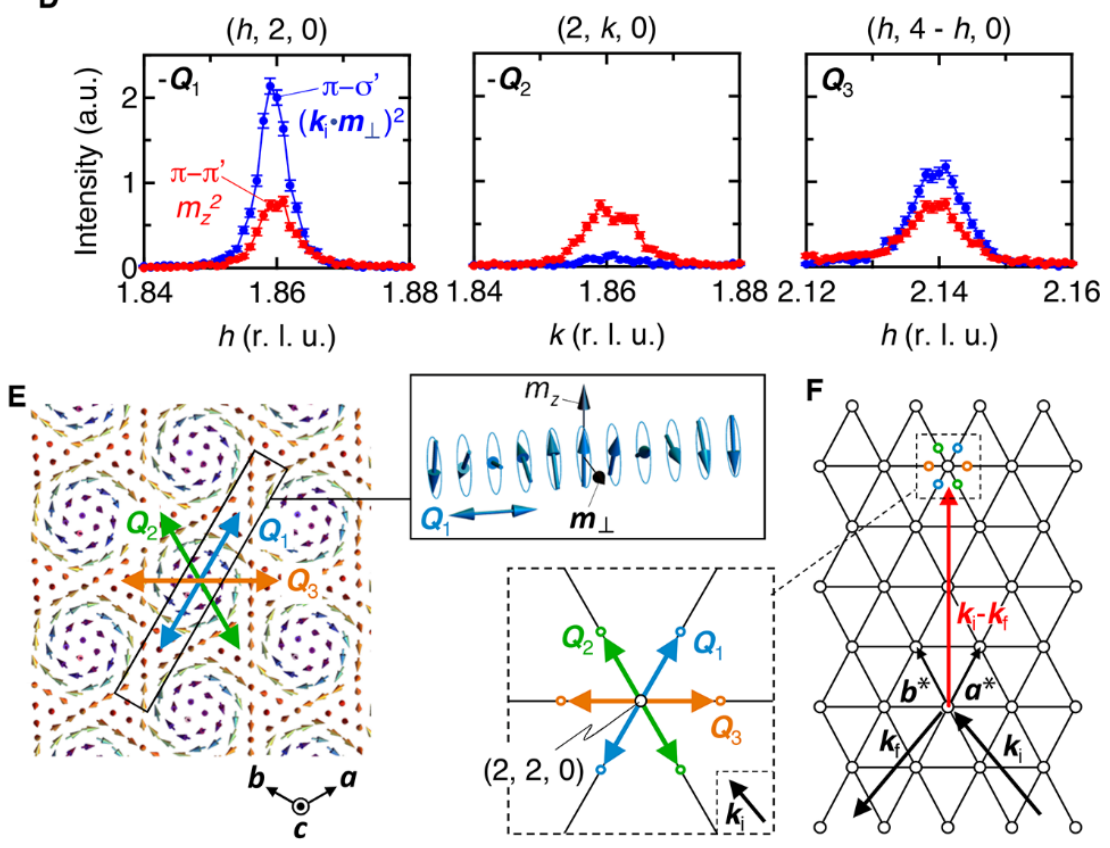

Fig. 3. Analysis of spin textures by resonant $x$-ray scattering. (A) $H$ dependence of $M$ in $H$-increasing (black dashed line) and decreasing (black solid line) sweeps and the difference between them ( $\triangle M$, purple solid line). (B) $q$, and (C) integrated intensity for each magnetic satellite peak at $Q_{i}(i=1,2,3)$ around the Bragg peak $(2,2$, 0 ), measured at $5 \mathrm{~K}$ and in an $\mathrm{H}$-decreasing sweep. (D) The intensity profile of magnetic reflection of each polarization channel for each (-) $Q$ i at $5 \mathrm{~K}$ with $H \| c$ of $7 \mathrm{kOe}$ in the $A(\mathrm{SkL}$ ) phase region. Red (blue) circle is for the $\Pi-\Pi^{\prime}\left(\Pi-\sigma^{\prime}\right)$ channel, which is approximately proportional to the $m_{z}^{2}\left(\left(\boldsymbol{k}_{\mathrm{i}} \cdot \boldsymbol{m}_{\perp}\right)^{2}\right)(39)$. $\Pi(\sigma)$ corresponds to the $x$-ray polarization parallel (perpendicular) to the $(0, O, L)$ plane, respectively. (E) Schematic real space texture for the Bloch-type SkL state with the definition of $Q_{i}(i=1,2,3)$. Inset: proper-screw type modulation component propagating along $Q_{1} . m_{z}$ and $\boldsymbol{m}_{\perp}$ represent the respective $c$-axis and ab-plane components of the magnetic moments. (F) The illustration of $x$-ray scattering condition in the reciprocal space. The inset is the magnified view around the $(2,2,0)$ indicating the relationship between $Q_{i}$ and $\boldsymbol{k}_{\text {i }}$. 